\title{
Avaliação de depressão em gestantes de alto-risco em um grupo de acompanhamento
}

\author{
Adriana Said Daher Baptista \\ Centro Universitário Hermínio Ometto, Araras-SP \\ Makilim Nunes Baptista \\ Universidade São Francisco, Itatiba-SP
}

\begin{abstract}
RESUMO
O presente estudo objetivou avaliar a sintomatologia depressiva em gestantes de alto-risco que participavam de um Grupo de Acompanhamento de Gestantes de Risco (GAGER) e avaliar a variação de sintomatologia em quatro momentos distintos. Foram participantes desta pesquisa, seis gestantes de alto-risco, de um Hospital-escola, avaliadas antes de participarem do grupo; após participarem por duas vezes do mesmo; 24 a 36 horas pós-parto e, quatro semanas após o parto. Foram utilizados como instrumentos entrevista clínica psicológica e a Escala de Depressão Pós-parto de Edimburgo. Os resultados demonstraram que apenas entre a $1^{\mathrm{a}}$ e $2^{\mathrm{a}}$ medidas encontrou-se diferença estatisticamente significante, além do que $50 \%$ das mulheres que apresentavam sintomatologia depressiva durante a gestação continuaram com a sintomatologia pós-parto. As limitações metodológicas do estudo, hipóteses sobre a não-diminuição da sintomatologia depressiva durante o acompanhamento e explanações da relação entre sintomatologia depressiva e períodos gravídicos e puerperais são abordados na discussão.
\end{abstract}

Palavras-chave: depressão; gestação; puerpério.

\section{ABSTRACT \\ Depression evaluation in an attendance group for high-risk pregnant women}

The goal of this study was to verify the variation of depression symptomatology in an informative high-risk post-partum group of pregnant (GAGER). Six high-risk pregnant women, from a University Hospital participated in this research, and they were evaluated four times: first, before forming the group; second, after two participations in this group; third, 24 to 36 hours after partum; and, four weeks post-partum. The instruments used were a Psychological Clinic Interview and, the Edinburgh Post-partum Depression Scale. Results showed that only between the first and second measure was observed significant statistic differences and approximately half of women that had depression during pregnancy maintained this symptomatology after pregnancy. Methodological limitations, hypothesis about non-modification in depressive symptomatology during the attendance and explanations about depression and pregnant and puerperal periods are discussed.

Keywords: depression; pregnancy; post-partum.

\section{INTRODUÇÃO}

A gravidez pode ser considerada um período de grandes mudanças para a vida da mulher e da família, sendo que, ao ocorrer intercorrência, de ordem materna ou fetal, passa a ser uma gravidez de alto-risco, que significa uma evolução desfavorável para o concepto e/ou para gestante, que afetará adversamente a gravidez e as pessoas que dela participam (Brazelton, 1988; Daher, Baptista \& Dias, 1999; Montenegro \& Rezende, 1987; Queenen, 1987). Algumas patologias, durante a gravidez, fazem com que a gestação se torne de altorisco, levando a gestante a acompanhamentos médicos, hospitalizações freqüentes e a sofrer procedimentos agressivos, sendo algumas delas: Trabalho de Parto Prematuro (TPP); Placenta Prévia; Deslocamento Prematuro da Placenta (DPP); Amniorrexe
Prematura; Diabetes Gestacionais (DG) e a Doença Hipertensiva Específica da Gravidez (DHEG).

Durante a gravidez observam-se diversas mudanças fisiológicas como, por exemplo, a variação hormonal, o que pode trazer mudanças orgânicas e comportamentais significativas, inclusive o desencadeamento ou exacerbação de sintomatologia depressiva (Baptista, Baptista \& Oliveira, 2004; Barini, 1997). Em relação aos sintomas depressivos na gravidez, pode-se observar que muitos destes, como ansiedade, baixa concentração, irritabilidade, fadiga, mudança no apetite, insônia ou hipersônia e perda de energia, são comuns também aos sintomas gestacionais e puerperais, o que dificulta discriminar se os sintomas apresentados fazem parte de um quadro gravídico ou depressivo. Outro fato importante é que a maioria dos estudos de depressão em gestantes aponta que não existe uma diferenciação da sintomatologia depressiva 
quando comparado a mulheres não gestantes, o que não denota um novo fenômeno psicopatológico para a gravidez (Alvorado \& cols., 1993; Coverdale, Mccullough, Chervernak \& Bayer, 1996).

A preocupação com o aspecto físico e diminuição do desejo sexual são também considerados como fazendo parte dos quadros depressivos e da gestação (Coverdale \& cols., 1996; Kennerley \& Gath, 1989). Assim, os dados obtidos com esses estudos, também dificultam o achado da prevalência da depressão durante a gravidez, já que muitos dos sintomas se sobrepõem (Zuckerman, Amaro, Bauchner \& Cabral, 1989). No entanto, há estudos que constatam uma frequiência maior de sintomatologia depressiva no intercurso da gravidez, como o de Alvorado e cols. (1993), em que observaram a prevalência de sintomas depressivos em grávidas e relacionaram estes com alguns fatores de risco, como por exemplo, gravidez indesejada, insatisfação na relação entre o casal e antecedentes pessoais de depressão. Outros fatores de risco associados à depressão durante a gravidez são: grande número de eventos vitais negativos durante esse período, baixo apoio emocional, solidão, menoridade, ser solteira, menor nível educacional, menor nível socioeconômico, antecedentes de aborto induzido e histórias de partos problemáticos anteriores (Gotlib, Whiffen \& Mount, 1989; Kumar \& Mordecai, 1984; Millán, 1990; Zuckerman \& cols., 1989).

A depressão pós-parto é um distúrbio prevalente, porém diferentes pesquisas apontam freqüências diversas desse problema na população. Talvez esse fato se dê devido ao uso de diferentes metodologias, instrumentos e por serem aplicados em amostras heterogêneas, podendo variar de 6,8\% a $30 \%$ (Cooper, Campbell \& Day, 1988; Cox, Murry \& Chapman, 1993; Kazdin, 1990; Kumar, 1990; Paykel, Emms, Fletcher \& Rassaby, 1980; Pop, Essed, Geus, Son \& Komproe, 1993; Warner, Appleby, Whitton \& Faragcher, 1996; Weissman \& Olfson, 1995; Whiffen, 1988).

Além de divergências na prevalência, muitos estudos demonstram uma variação quanto ao momento do surgimento da depressão pós-parto, girando em torno de duas semanas para Weissman e Olfson (1995); quatro semanas (APA, 1994); cinco semanas (Cox \& cols., 1993); seis semanas (CID, 1993; Hopkins, Campbell \& Marcus, 1989); de seis a oito semanas (Warner \& cols., 1996); dez semanas (Pop \& cols., 1993); de dois a três meses (Kumar, 1990), o que demonstra, que esse fenômeno deve ser melhor estudado e conceituado, já que a variação de tempo de ocorrência é muito grande entre os que o analisa.

Com o advento da entrada do psicólogo hospitalar no contexto de saúde, vêm se observando progressivamente a utilização de programas terapêuticos, de apoio, intervenção e acompanhamento em pacientes com diversos problemas de saúde, em todos os níveis do Sistema Único de Saúde, principalmente grupos de apoio ambulatoriais e em enfermaria (Baptista \& Dias, 2003).

A psicoterapia de grupo, na década de 50 passou a ser amplamente utilizada na área da saúde, em hospitais gerais e psiquiátricos, direcionando-se a programas de reabilitação. Na década de 70 , passou-se a exigir um enfoque nas modalidades de grupos (crianças, adultos, pacientes em ambientes médicos, entre outros) e do prazo de tempo curto para o tratamento diante das necessidades sociais e econômicas da época (Scheidlinger, 1996). Já no início do século XX, o uso da psicoterapia de grupo, para o tratamento de enfermidades físicas, era realizado tanto de modo didático, na qual o paciente recebia informações sobre a doença, quanto de modo participativo, quando compartilhavam experiências e sentimentos. Atualmente, além da utilização de técnicas de controle de estresse, têmse integrado nos tratamentos de pacientes enfermos fisicamente, os métodos didáticos, informativos e participativos (Ulman, 1996).

Com o desenvolvimento das modalidades, nas abordagens da saúde geral, pode-se observar dois modos de tratamento em grupo: grupo psicoterápico e o de consultoria, sendo, o segundo, mais utilizado. Este último é formado em conjunto com as equipes médicas, no qual os pacientes aprendem a lidar com seus problemas médicos e psicológicos específicos. Dezadorlf e Bilar (1995) propõem uma intervenção, na qual o enfoque é a informação educacional, para tanto, utilizam informações de procedimentos (referentes ao detalhamento dos aspectos de cada procedimento médico) e informações das sensações (sensações prováveis que o paciente possa sentir antes, durante e após as intervenções médicas, preparando o paciente e auxiliando em estratégias de enfrentamento), objetivando a adesão do paciente ao tratamento e consentimento às intervenções e procedimentos médicos. Já Courchaine e Dowd (1995) propõem um modelo cognitivo-comportamental de intervenção grupal com pacientes internados ou ambulatoriais em crise, abordando a problemática, com o objetivo dos participantes aprenderem a lidar com o fator perturbador, permitindo o indivíduo retomar suas atividades de maneira funcional.

Dezadorlf e Bilar (1995) afirmam que os problemas dos pacientes hospitalizados devem ser tratados de forma biopsicossocial, pois geralmente estas possuem um déficit significativo no suporte emocional. Por isso, os autores propõem intervenções em diversas áreas, como na área biológica (controle da patologia através do tratamento); afetiva (discutir o enfrentamento dos estados de ansiedade, de depressão e hostilidade); cognitiva (providenciar informações ao paciente em um processo psicoeducacional, com o propósito de compreender seu problema, facilitando a adaptação e enfrentamento) e comportamental (desenvolver um 
programa de modificação do comportamento problema, discriminando os fatores de risco e treinar o paciente para automonitoramento aumentando a habilidade de autocuidado).

Os grupos de informação parecem ser uma das estratégias mais eficazes em instituições de saúde, já que podem propiciar uma intervenção com custo baixo, atendendo um grande número de pacientes (devido à grande demanda na área da saúde, principalmente nos países em desenvolvimento) e levando-se em conta a insuficiência de profissionais na área de saúde.

Sendo assim, o objetivo desta pesquisa foi verificar se o Grupo de Acompanhamento de Gestantes de Risco (GAGER) auxilia na diminuição da sintomatologia depressiva.

\section{METODOLOGIA}

\section{Participantes}

A pesquisa foi realizada com seis gestantes de altorisco, internadas numa Enfermaria de Obstetrícia de um Hospital-Escola de Campinas - São Paulo, sendo que nenhuma das gestantes estava em acompanhamento psiquiátrico ou já tinha apresentado algum transtorno psiquiátrico, que necessitou de hospitalização anteriormente.

Inicialmente participaram 42 mulheres, sendo que somente seis concluíram as quatro medidas. Esta perda se deu a diversos fatores, como por exemplo, algumas gestantes não realizaram o parto neste Hospital, sendo inviável as demais avaliações ou dificuldade em encontrar o endereço das gestantes, pois a maioria morava em locais não cadastrados pela Prefeitura.

Os seis sujeitos que obtiveram todas as medidas, tinham em média ( \pm DP) $22,7 \pm 4$ anos, com idade entre 16 e 27 anos, residiam na cidade de Campinas - São Paulo e foram atendidos pelo Sistema Único de Saúde (SUS).

Em relação aos sujeitos, $50 \%$ estava trabalhando e $50 \%$ era "do lar". Sobre a escolaridade, $66,6 \%$ possuía o $1^{\circ}$ grau incompleto, seguidos de $16,6 \%$ com o primeiro grau completo e a mesma porcentagem com o $2^{\circ}$ grau incompleto. A renda familiar da maioria da amostra $(83,3 \%)$ era de até seis salários mínimos. O estado civil da amostra se dividiu em $50 \%$ de casadas, $33,3 \%$ de solteiras e $16,6 \%$ de amasiadas. A metade da amostra era primípara $(50 \%)$ e o restante se dividia entre a segunda gestação e acima de três gestações, sendo que nenhuma havia abortado anteriormente. A maioria dos sujeitos planejou a gravidez $(66,6 \%)$ e, a totalidade havia realizado o exame pré-natal. A respeito das patologias apresentadas pela amostra, $66,6 \%$ possuíam DHEG, 16,6\% Trombose Venosa Profunda e $16,6 \%$ TPP.

\section{Material}

Protocolo das Gestantes de Alto-risco - constituiu-se de um protocolo que visa a caracterização de dados sociodemográficos, genetograma, história gestacional, informações sobre a história pessoal e familiar psiquiátrica, histórico de outras gravidezes, dados de observações clínicas, sociais e/ou psicológicas relevantes à internação.

Escala de Depressão Pós-parto de Edimburgo escala de auto-administração composta de 10 itens, elaborada por Cox e cols. (1987), sendo a nota de corte utilizada de 12 pontos, para separar as mulheres que estavam apresentando a sintomatologia depressiva relevante. Essa nota foi sugerida pelo estudo, através do qual ocorreu a validação realizada no Brasil, no Distrito Federal - Brasília, por Santos (1995), em sua dissertação de mestrado. A Escala de Depressão PósParto de Edimburgo é uma escala que avalia os sintomas depressivos numa graduação de quatro pontos, apresentando sensibilidade de $86 \%$, especificidade de $78 \%$ e valor preditivo positivo de $73 \%$. A fidedignidade alcançada pelo método das duas metades foi 0,88 e o coeficiente alfa estandardizado encontrado foi 0,87 .

\section{Procedimento}

A primeira medida ocorreu no pré-parto e antes dos sujeitos participarem do GAGER. A segunda medida também ocorreu no pré-parto e após os sujeitos terem participado pelo menos duas vezes do GAGER. A terceira medida ocorreu de vinte e quatro a trinta e seis horas após o parto e, a quarta medida ocorreu quatro semanas após o parto.

As avaliações psicológicas pré e a primeira no pósgrupo ocorreram nos leitos das pacientes e a intervenção grupal foi realizada na Enfermaria de Obstetrícia de um Hospital-Escola, sempre nos quartos onde existia o maior número de gestantes em repouso, isto é, que estavam impossibilitadas de se locomoverem ou ficarem sentadas, durante a realização da reunião grupal.

As avaliações psicológicas, que ocorreram quatro semanas após o parto, foram realizadas na casa das puérperas, através de visitas domiciliares.

Os profissionais envolvidos nesta pesquisa foram: uma psicóloga, responsável pela entrevista psicológica, pelas avaliações psicológicas pré e pós grupo e pela condução das atividades grupais com as gestantes, abordando os aspectos psicológicos; uma assistente de pesquisa; uma enfermeira e um médico residente (membros do Grupo Informativo). 
Após a avaliação pré-grupo, os sujeitos foram submetidos a um grupo informativo e instrutivo (GAGER), através do qual receberam informações, puderam refletir e discutir os aspectos de saúde, sociais, psicológicos e patológicos da gestação (Daher, 1999).

\section{Conteúdo do GAGER}

Os tópicos abordados no GAGER partiram de observações na enfermaria de obstetrícia e Unidade de Terapia Intensiva Neonatal (UTI-Neo), além de diversos levantamentos por questionário, com perguntas fechadas e abertas, realizado com as gestantes, no próprio setor. Os temas abordados no grupo foram:

Aspectos de Saúde - sinais e sintomas comuns ao final da gestação, sinais de trabalho de parto, tipos de parto, alojamento conjunto e berçário, UTI Neo e amamentação.

Aspectos Sociais - informações sobre direitos e deveres com relação à licença maternidade, licença paternidade, afastamento por doenças e registro de nascimento.

Aspectos Psicológicos - os aspectos psicológicos foram abordados durante todo processo do grupo, sendo que a psicóloga atuou observando as gestantes, durante a exposição dos outros profissionais e, ao perceber falta de compreensão por parte das participantes, atuou no sentido de ajudá-las a enfrentarem suas dúvidas. Dentro do processo, as gestantes eram motivadas a falar sobre suas crenças populares, seus medos e expectativas, sentimentos acerca do seu estado de saúde e hospitalização, o relacionamento com a equipe de saúde e companheiras de quarto, além do relacionamento familiar.

Aspectos Médicos - os médicos residentes eram convidados, ao final do grupo, a responderem aos questionamentos levantados, durante o andamento do grupo.

\section{RESULTADOS}

A análise dos dados referentes à sintomatologia depressiva foi realizada por meio da comparação dos resultados da Escala de Depressão de Edimburgo PósParto nas quatro etapas do estudo, pela prova de Wilcoxon para medidas relacionadas (nível de significância de 0.05). Para a análise, foi utilizado o Programa SPSS (Statistical Package for Social Sciences, versão 8.0), para Windows.

Na tabela 1 estão os resultados obtidos nas quatro avaliações, sendo que somente foi observada diferença estatisticamente significante entre a primeira e a segunda avaliação. Embora na quarta avaliação tenha sido observada a maior média entre as medidas, também houve uma variabilidade grande que inviabiliza a localização de outras diferenças estatísticas.

Tabela 1: Comparação dos resultados da Escala de Depressão Pós-Parto de Edimburgo nas quatro medidas

\begin{tabular}{cc|cccccc}
\hline \multirow{3}{*}{ Medidas } & Média $( \pm \mathrm{DP})$ & $1 \times 2$ & $1 \times 3$ & $1 \times 4$ & $2 \times 3$ & $2 \times 4$ \\
\cline { 3 - 7 } & $8,3 \pm 4,5$ & & & & & \\
& & & & & \\
2 & & $\mathrm{Z}=-2,1 ; \mathrm{p}=$ & $\mathrm{Z}=-0,1 ; \mathrm{p}=$ & $\mathrm{Z}=-0,8 ; \mathrm{p}=$ & $\mathrm{Z}=-1,4 ; \mathrm{p}=$ & $\mathrm{Z}=-0,1 ; \mathrm{p}=$ & $\mathrm{Z}=-0,9 ; \mathrm{p}=$ \\
3 & $10,8 \pm 5,5$ & 0,039 & 0,92 & 0,40 & 0,18 & 0,92 & 0,34 \\
4 & $8,8 \pm 5,4$ & & & & & \\
\hline
\end{tabular}

A Tabela 2 contém os resultados obtidos pela amostra final, isto é, as seis pacientes que tiveram as quatro medidas. Nota-se que os sujeitos quatro e seis que apresentaram pontuação significativa de depressão na primeira medida, mantiveram a mesma classificação na segunda e quarta medidas, sendo que a gestante quatro mostrou-se com pontuação significativa também na terceira medida. $\mathrm{O}$ sujeito um mostrou baixa pontuação em sintomatologia depressiva durante todo o processo, ultrapassando o ponto de corte no pós-parto; o sujeito dois pontuou acima do corte após a participação no grupo e, posteriormente demonstrou diminuição gradativa nas duas outras medidas. Os sujeitos três e cinco não apresentaram sintomatologia depressiva durante todo o processo.

Tabela 2: Comparação dos sujeitos nas quatro aplicações da Escala de Depressão Pós-Parto de Edimburgo

\begin{tabular}{|c|c|c|c|c|}
\hline Sujeito & 1 Medida & 2 Medidas & 3 Medidas & 4 Medidas \\
\hline
\end{tabular}




\begin{tabular}{|c|c|c|c|c|c|c|c|c|}
\hline & \multicolumn{2}{|c|}{$(\mathrm{N}=42)$} & \multicolumn{2}{|c|}{$(\mathrm{N}=16)$} & \multicolumn{2}{|c|}{$(\mathrm{N}=11)$} & \multicolumn{2}{|c|}{$(\mathrm{N}=06)$} \\
\hline & Valor & Decisão & Valor & Decisão & Valor & Decisão & Valor & Decisão \\
\hline 01 & 4 & -- & 4 & -- & 8 & -- & 20 & Depressiva \\
\hline 02 & 7 & -- & 12 & Depressiva & 6 & -- & 3 & -- \\
\hline 03 & 5 & -- & 6 & -- & 3 & -- & 9 & -- \\
\hline 04 & 15 & Depressiva & 18 & Depressiva & 18 & Depressiva & 16 & Depressiva \\
\hline 05 & 6 & -- & 9 & -- & 5 & -- & 1 & -- \\
\hline 06 & 13 & Depressiva & 16 & Depressiva & 11 & -- & 21 & Depressiva \\
\hline
\end{tabular}

\section{DISCUSSÃO}

Inicialmente, é importante frisar, que o instrumento utilizado para avaliar a sintomatologia depressiva pode ter algumas limitações. A validação do instrumento e a indicação do ponto de corte foram realizadas com uma amostra de sujeitos de nível socioeconômico médio/alto (Santos, 1995). Talvez, pelas peculiaridades da amostra deste estudo, por se tratar de sujeitos de nível socioeconômico baixo, ter baixa escolaridade e serem gestantes de alto-risco, o ponto de corte possa não expressar, de forma consistente, a decisão de terem a sintomatologia depressiva ou não.

Nesse sentido, não há uma escala apropriada para a população de gestantes de alto-risco (Coverdale \& cols., 1996). A utilização desse instrumento foi preferido devido a sua validação ter sido realizada com a população de puérperas brasileiras; ser de fácil aplicação e correção e ser breve, além da linguagem acessível.

Não foi utilizada a denominação depressiva para classificar as condições do humor das participantes deste estudo, e sim o termo sintomatologia depressiva. Essa decisão ocorreu em função de não se ter um diagnóstico, utilizando-se de avaliações psiquiátricas e psicológicas completas. Portanto, os dados coletados não são suficientes para diagnosticar um estado psicopatológico (APA, 1994).

Não foi utilizado nenhum exame laboratorial para verificar o nível hormonal dos sujeitos no pós-parto. Desta forma, não se pode discutir tal indicativo para o desencadeamento da depressão pós-parto como é proposto por alguns estudiosos (Cooper \& Stein, 1989; Martin, Brown, Goldberg \& Brockington, 1989; O'Hara, Nennaber \& Zekosky, 1984; Paykel \& cols., 1980).

É importante ressaltar, que o tamanho da amostra utilizada neste estudo não pode ser considerada ideal para generalizações dos dados com parturientes de diferentes camadas sociais e/ou condições socioeconômicas adversas.

Em relação à perda amostral, já explicada na metodologia, dificuldades institucionais e da política de saúde auxiliaram para que isso ocorresse. Um outro grande problema foi encontrar a residência dessas parturientes para a $4^{\mathrm{a}}$ testagem, já que na maioria das vezes, as próprias gestantes não sabiam especificar o local da residência ou davam dados incorretos, além do que a maioria das residências se encontrava na periferia da cidade, onde nem sempre os dados residenciais estavam catalogados nos mapas da cidade.

Um aspecto de relevância refere-se ao uso de medicamentos calmantes pela maioria das gestantes de alto-risco, durante a hospitalização, em função de terem que ficar restritas ao leito por um período indeterminado, o que pode ter afetado as medições, bem como os resultados dos dados. Esse procedimento médico é padrão e não se sabe bem ao certo quais são as interferências exatas desse procedimento em relação ao humor das gestantes. Talvez, algumas das consequiências dos calmantes, possam interferir na mensuração dos sintomas depressivos, principalmente em relação ao rebaixamento das funções físicas, menor frequiência de relatos verbais e possíveis modificações nas associações cognitivas, entre outros (Graeff, 1989; p. 64).

Não foi encontrado na literatura algum estudo relatando o nível de sintomatologia depressiva em gestantes de alto-risco e, nem mesmo, o nível de depressão pós-parto de puérperas que tiveram uma gestação com intercorrências. Lanczik e cols. (1992), quando estudaram as sintomatologias presentes no "blues" utilizaram como fator de exclusão em sua amostra, as gestantes que apresentaram complicações gestacionais. Talvez, por essa população apresentar alguns sinais e sintomas das intercorrências da gestação, parecidos com a sintomatologia depressiva, o diagnóstico de depressão venha a ser de difícil caracterização (Zuckerman \& cols., 1989).

Todas essas características podem ter confundido a análise da sintomatologia depressiva em gestantes de alto-risco, mesmo porque o próprio conceito de "altorisco" não possui uma definição única e coerente entre os diversos autores (Sussman, Simon, Glynn \& Stacy, 1996). A utilização do termo alto-risco, aqui utilizado, denomina o estado de saúde física da gestante e/ou do concepto e não em relação às condições psicológicas e psiquiátricas da gestante.

Outro fator que merece destaque, diz respeito aos momentos da aplicação dos instrumentos utilizados na coleta de dados e as grandes mudanças que ocorrem durante a gestação, parto e puerpério. A primeira aplicação ocorreu no início da hospitalização das gestantes de alto-risco na enfermaria, podendo significar, que o nível de tolerância quanto ao ficar longe de suas 
famílias, de seu trabalho, de suas atividades diárias e suas relações sociais, ainda estivesse alto, pois o tempo de internação ainda era pequeno.

$\mathrm{Na}$ segunda medida, as gestantes já estavam hospitalizadas por no mínimo dois dias, isto é, em adaptação com a rotina e procedimentos hospitalares. Já na terceira medida, tinham passado pelo parto e estavam vivenciando as consequiências de uma gestação de alto-risco, com o nascimento do bebê. Pôr último, na quarta medida, as puérperas não se encontravam mais hospitalizadas e estavam cuidando de seus bebês. As variáveis presentes, nesse último momento, eram referentes às questões sociais, familiares e ambientais, as quais as puérperas teriam que se adaptar a um novo papel, principalmente as primíparas.

Pode-se observar, que as atividades profissionais da maioria dos sujeitos deste estudo se enquadravam em "do lar"; a renda familiar foi baixa e o nível de escolaridade também, o que se pode supor a existência de privação em fatores importantes como: alimentação, medicação, assistência à saúde, informação, entre outros e, estes fatores podem contribuir para o desenvolvimento da ocorrência da sintomatologia depressiva (Gotlib \& cols., 1989; Millán, 1990; Kumar \& Mordecai, 1984; Zuckerman \& cols., 1989).

Os fatores anteriormente citados podem ser fundamentais para tentar explicar o alto índice de sintomatologia depressiva encontrada nesta amostra (aproximadamente $50 \%$ na segunda e quarta medidas), apesar da amostra ser pequena. Estes resultados são incompatíveis com os estudos sobre depressão em gestantes e depressão pós-parto observados na literatura (Alvorado \& cols., 1993; Cooper \& cols., 1988; Cox \& cols., 1993; Kazdin, 1990; Kumar, 1990; Paykel \& cols., 1980; Pop \& cols., 1993; Warner \& cols., 1996; Weissman \& Olfson, 1995; Whiffen, 1988), em que a prevalência de depressão varia entre 6,8 a $30 \%$, sendo importante ressaltar, que nenhum desses estudos foram realizados com gestantes de alto-risco.

Ainda referente à prevalência de sintomatologia depressiva no pós-parto, Kaplan e Sadock (1997) explicam que 20 a $40 \%$ das mulheres podem apresentar alguma disfunção cognitiva ou emocional durante esse período, devido a várias condições dessa fase, como aumento da responsabilidade, diminuição das relações sexuais e menor atenção do esposo.

$\mathrm{Na}$ entrevista inicial (os sujeitos ainda eram gestantes), foi questionado aos sujeitos quanto a sentimentos de tristeza em pós-partos anteriores e $31 \%$ da amostra não pôde responder, pois se tratavam de primigestas. Mas a grande maioria das multíparas, em todas as medidas, referenciou não ter sentido tristeza. As respostas devem ser vistas com cautela, pois foram apoiadas na memória dos sujeitos e não realizada no momento do pós-parto anterior. A intenção era investigar a labilidade do humor logo após o parto, mas a pergunta referia-se apenas ao sentimento de tristeza e não a sentimentos disfóricos, presentes no "blues". Levando-se em conta a resposta de tristeza, os resultados encontrados não condizem com os da literatura, uma vez que Kumar (1990) relata que 50 a $75 \%$ das puérperas passam por mudança de humor.

A respeito da sintomatologia depressiva, perguntada às gestantes, muitos estudos relatam que o histórico pessoal sobre a experiência anterior da gestação de sintomas depressivos é um fator de grande importância na etiologia da depressão durante a gestação e no pós-parto. Quando se observa a resposta dos sujeitos, na primeira medida, enquanto gestantes, percebe-se que $90,5 \%$ delas responderam ter apresentado sintomatologia depressiva anterior e mostraram-se deprimidas no resultado do instrumento utilizado para tal avaliação (Escala de Depressão Pós-Parto de Edimburgo).

Esses resultados confirmam o estudo de Alvorado e cols. (1993), em que um dos fatores associado à depressão, durante a gestação, é ter antecedente pessoal de depressão. Em relação aos sujeitos com quatro medidas, percebe-se que todas as gestantes, que relataram apresentar as sintomatologias depressivas anteriores, mostraram-se puérperas deprimidas no pósparto, confirmando os achados de Paykel e cols. (1980), porém não confirmando totalmente os achados de Weissman e Olfson (1995), que relataram, em seu estudo, que 20 a $30 \%$ das grávidas com histórico de depressão anterior não manifestavam o transtorno.

Na comparação entre os resultados da sintomatologia depressiva durante as medidas deste estudo, algumas considerações devem ser feitas, a fim de levantar algumas hipóteses a respeito dos resultados encontrados, após o tratamento estatístico.

Talvez, a diferença entre a primeira e segunda medida tenha sido significante, pois uma das funções do grupo, segundo Dezadorlf e Bilar (1995), é fornecer informações sobre procedimentos e sensações que podem aparecer durante o processo, sendo que as pacientes podem entrar mais em contato com dados da realidade, o que muitas vezes, são contrárias a certas expectativas, como por exemplo, a gestante acreditar que o nenê vai nascer e ficar em alojamento conjunto, ou a gestante poderá ir para casa com o bebê, logo após o parto, o que não ocorre na maioria das vezes.

Através dos resultados obtidos sobre sintomatologia depressiva, mesmo tomando as precauções referentes ao tamanho da amostra, a Escala de Depressão Pós-Parto de Edimburgo pode dar indicativos, de que as mulheres que apresentaram sintomatologia depressiva durante a gestação, mostraram-se com a mesma característica após o parto, contrariando os achados de 
Appleby, Gregoire, Platz, Prince e Kumar (1994), os quais não associaram a escala de Edimburgo como prevendo essa relação anteparto e pós-parto.

Uma das hipóteses da não influência do GAGER na depressão pós-parto é o fato de que, aproximadamente 50\% da amostra já apresentava sintomatologia clinicamente significativa de depressão enquanto gestantes. Talvez, o sujeito que apresenta sintomatologia depressiva durante a gestação possua características diferenciadas no prognóstico da depressão pósparto, em relação à gestante que desenvolve depressão somente no pós-parto. Porém, se as gestantes que apresentavam sintomatologia depressiva fossem eliminadas da amostra, não seria possível realizar a análise estatística.

Talvez, a psicoterapia de grupo fosse mais aconselhada para as mulheres que já estavam apresentando sintomatologia depressiva durante a gestação, ou mesmo, o GAGER seja mais aconselhável para mulheres que nunca tiveram depressão anterior.

Uma outra possível explicação para a não influência do GAGER na depressão pós-parto refere-se ao número reduzido de sujeitos com as quatro medidas, o que pode ter explicado a não aceitação da hipótese da diferença. Talvez, com um número superior de sujeitos, os resultados poderiam ser diferentes dos encontrados nesta pesquisa, porém estratégias específicas deveriam ser pensadas em relação ao controle da perda amostral.

Uma sugestão para trabalhos posteriores é verificar a influência do GAGER nas estratégias de enfrentamento referentes à hospitalização, parto e puerpério, em gestantes de alto-risco, bem como nos níveis de ansiedade presentes nesses momentos. De qualquer forma, Thurtle (1995), em seu artigo, relata que a falta de uma definição homogênea, como também clareza na etiologia da depressão pós-parto, tanto no domínio biomédico quanto psicológico, revela a necessidade da atuação multidisciplinar para o atendimento dessas mulheres.

\section{REFERÊNCIAS}

Alvorado, R. R., Perucca, P. E., Neves, E., Rojas, M., Monardes, J., Olea, E. E. \& Vera, A. (1993). Cuadros Depressivos Surante El Embarazo y Fatctores Asociados. Revista Chilena de Obstetrícia y Ginecologia, 58(2), 135-141.

American Psychiatric Association (1994). Diagnostic and statistical manual of mental disorders $\left(4^{\text {th }}\right.$ ed.) Washington, DC [Manual Diagnóstico e Estatístico de Transtornos Mentais, 1995]. Tradução Dayse Batista. Porto Alegre: Artes Médicas.

Appleby, L., Gregoire, A., Platz, C., Prince, M. E. \& Kumar, R. (1994). Screening women for high risk of postnatal depression. Journal of Psychosomatic Research, 38(6), 530-545.

Baptista, M. N. \& Dias, R. R. (2003). Psicologia hospitalar: teoria, aplicações e casos clínicos. Rio de Janeiro: GuanabaraKoogan.
Baptista, M. N., Baptista, A. S. D. \& Oliveira, M. G. (2004), Depressão e Gênero: por que as mulheres deprimem mais que os homens? Em M. N. Baptista (Org.), Suicídio e depressão: atualizações (pp. 50-61). Rio de Janeiro: Guanabara-Koogan.

Barini, R. (1997). Modificações hormonais e variações comportamentais na mulher. Em D. R. Zamignani. Sobre comportamento e cognição V. 3. (pp. 41-48). Santo André: ARBytes.

Brazelton, T. B. (1988). O desenvolvimento do apego: uma família em formação. Porto Alegre: Artes Médicas.

Classificação dos transtornos mentais e de comportamento da CID - 10: descrições clínicas e diretrizes diagnósticas. Organização Mundial de Saúde/Genebra. Porto Alegre: Artes Médicas.

Cooper, P. J. \& Stein, A. (1989). Life events and postnatal depression: the Oxford study. Em Cox, J. L., Paykel, E. S \& Page, M.L. Childbirth as a life event (pp. 123-135) Southampton: Duphar Medical Relations.

Cooper, P. J., Campbell, E. A. \& Day A. (1988). Nonpsychotic psychiatric disorder after childbirth: a prospective study of prevalence, incidence, course and nature. British Journal Psychiatry, 152, 799-806.

Courchaine, K. E \& Dowd, E. T. (1995). Abordagens de grupo. Em F. M. Dattílio \& A. Freeman (Orgs.), Estratégias cognitivo-comportamentais para intervenção em crises. V. II (pp. 7593). São Paulo: Editorial PsyII.

Coverdale, J. H., Mccullough, L. B., Chervernak F. A. \& Bayer, T. (1996). Clinical implications and management strategies when depression occurs during pregnancy. Australin New Zeland Journal Obstetric and Gynecology, 36(4), 424-429.

Cox, J. L., Murry, D. \& Chapman, G. (1993). A controlled study of the onset, duration and prevalence of postnatal depression. British Journal of Psychiatry, 163, 27-31.

Daher, A. S. (1999). Grupo informativo às gestantes de alto-risco: influência na depressão pós-parto. Dissertação de Mestrado não publicada da PUCCAMP. Campinas, SP.

Daher, A. S., Baptista, M. N. \& Dias, R. R. (1999). Aspectos relevantes da depressão na gravidez e dos transtornos pósparto. PSICOUSF, 4(1), 77-92.

Dezadorlf, W. W. \& Bilar, C. D. (1995). Intervention strategies in clinical health psychology. Em W. W. Dezadorlf \& C. D. Bilar Clinical health psychology in medical setting practitioner's guidebook (pp. 69-100). British Library Calologing in Publication Date.

Gotlib, I. H., Whiffen, V. E. \& Mount, J. H. (1989). Prevalence rates and demographic characteristic associated with depression in pregnancy and postpartum. Journal of Consulting and Clinical Psychology, 57, 269-274.

Graeff, F. G. (1989). Drogas Psicotrópicas e seu modo de ação. São Paulo: E P U.

Hopkins, J., Campbell, S. B \& Marcus, M. (1989). Postpartum depression and postpartum adaptation: overlapping constructs? Journal of Affective Disorders, 17, 251-254.

Kaplan, H. I. \& Sadock B. J. (1997). Compêndio de psiquiatria. Trad. Dayse Batista. Porto Alegre: Artes Médicas.

Kazdin, A. E. (1990). Childhood depression. Journal of child Psychology and Psychiatry, 31(1), 121-160.

Keneerley, H. \& Gath, D. (1989). Maternity blues: detection and measurement by questionnaire. British Journal of Psychiatry, 149, 240-243.

Kumar, R. \& Mordecai, R. K. (1984). A prospective study of emotional disorders in childbearing women. British Journal of Psychiatry, 144, 35-47. 
Kumar, R. (1990). Childbirth and mental illness. Triangle, 29(2/3), 73-81.

Lanczick, M., Spingler, H., Heidrich, A., Becker, T., Kretzer, B., Albert, P. \& Fritze, J. (1992). Postpartum "blues": depressive disease a pseudoneurasthenic syndrome. Journal of Affective Disorders, 25, 47-52.

Martin, C. J., Brown, G. W., Goldberg, D. P \& Brockington, I. F. (1989). Psyco-social stress and puerperal depression. Journal of Affective Disorders, 16, 283-293.

Millán, T. (1990). Encuesta sobre síntomas de depresión en embarazadas de um consultorio urbano de atención primaria. Revista Médica Chilena, 118, 1230-1234.

Montenegro, C. A. B. \& Rezende, J. (1987). Obstetrícia Fundamental. ( $5^{\mathrm{a}}$ ed.). Rio de Janeiro: Editora Guanabara.

O'hara, M. W., Nennaber, D. J. \& Zekoski, E. M. (1984). Prospective study of postpartum depression: prevalence, course and predictive factors. Journal of Abnormal Psychology, 93, 158-178.

Paykel, E. S., Emms, E. M., Fletcher, J. \& Rassaby, E. S. (1980). Life events and social support in puerperal depression. British Journal Psychiatry, 136, 339-346.

Pop, V. J. M., Essed, G. G. M., Geus C. A.; Son, M. M. \& Komproe, I. H. (1993). Prevalence of post partum depression or is it post-puerperium depression? Acta Obstetetricia e Gynecologia Scandinavica 2 72, 354-358.

Queenen, J. (1987). Gravidez de alto risco. (2 ${ }^{\mathrm{a}}$ ed.). São Paulo: Manole.

Santos, M. F. S. (1995). Depressão no pós-parto: validação da Escala de Edimburgo em puérperas brasilienses. Dissertação de Mestrado, Universidade de Brasília. Brasília, BR.
Scheidlinger, S. (1996). História da psicoterapia de grupo. Em H. I. Kaplan \& B. J. Sadock (Orgs.), Compêndio de psicoterapia de grupo ( $3^{\mathrm{a}}$ ed.) (pp. 6-13). Porto Alegre: Artes Médicas.

Sussman, S., Simon, T. R., Glynn, S. M. \& Stacy, A. W. (1996). What does "High Risk" mean? Behavior Therapy, 27, 53-65.

Thurtle, V. (1995). Post-natal depression: the relevance of sociological approaches. Journal of Advanced Nursing, 22, 416-424.

Ulman, K. H. (1996). Psicoterapia de grupo com os fisicamente enfermos. Em H. I. Kaplan \& B. J. Sadock (Orgs.), Compêndio de psicoterapia de grupo ( $3^{\mathrm{a}}$ ed.) (pp. 382-390). Porto Alegre: Artes Médicas.

Warner, R., Appleby, L., Whitton, A. \& Faragcher, B. (1996). Demographic and obstetric risk factories for post natal psychiatric morbidity. British Journal of Psychiatry, 168, 607-611.

Weissman, M. M. \& Olfson, M. (1995). Depression in women: implication for health care research. Science, 269(5225), 799801.

Whiffen, V. E. (1988). Vulnerability to postpartum depression: a prospective multivariated study. Journal of Abnormal Psychology, 97(4), 467-474.

Zuckerman, B., Amaro, H., Bauchner, H. \& Cabral, H. (1989). Depressive symptoms during pregnancy: relationships to poor health behaviors. American Journal Obstetric and Gynecologia, 160, 1107-1111.

Enviado: 31/08/2004 Revisado: $20 / 06 / 2006$ Aceito: $25 / 06 / 2005$

\section{Nota:}

${ }^{1}$ Pesquisa financiada pelo CAPES.

\section{Sobre os autores:}

Adriana Said Daher Baptista: Departamento de Psicologia da Fundação Hermínio Ometto - Uniararas - Endereço eletrônico: daherbaptista@ig.com.br.

Makilim Nunes Baptista: Programa de Pós-Graduação Strictu Senso em Psicologia da Universidade São Francisco (USF)- Itatiba/SP. 